\title{
ON THE CONJUGATE POINTS OF FOURTH ORDER, SELFADJOINT LINEAR DIFFERENTIAL EQUATIONS
}

\author{
G. J. ETGEN AND J. B. SCOTT
}

ABstract. In a fundamental paper, W. Leighton and Z. Nehari studied the oscillation of solutions of fourth order, selfadjoint differential equations. One of their principal results is a characterization of the conjugate points. In this characterization, however, the proof of the essential uniqueness of the solutions determining the conjugate points is not complete. The purpose of this note is to complete their proof thereby establishing the essential uniqueness of the solutions involved in characterizing the conjugate points.

In a fundamental paper, W. Leighton and Z. Nehari [1] studied the fourth order equation

$$
\left[r(x) y^{\prime \prime}\right]^{\prime \prime}-p(x) y=0
$$

where $r(x)$ and $p(x)$ are positive continuous functions on $[a, \infty)$. We note here that the proof of Theorem 3.6 of this paper is not complete in the sense that the essential uniqueness (i.e., unique up to constant multiples) of the solutions determining the points conjugate to $x=a$ has not been established. We illustrate our point. Suppose $y(x)$ is a solution of (1) having exactly $n+3$ zeros, $n \geqq 1$, on $[a, b]$ with the zeros at $x=a$ and at $x=b$ being double. Then, according to Theorem 3.6 (the numbered theorems and lemmas refer to those in [1]), the point $x=b$ is the $n$th conjugate point of $x=a$. However, we claim that it must also be shown that there cannot exist a solution $z(x)$ of (1) having double zeros at $x=a$ and $x=\beta, \beta<b$, with exactly $n+3$ zeros on $[a, \beta]$. In addition, if $y(x)$ is a solution of (1) having $n+3$ zeros on $[a, b]$ with the zero at $x=a$ being double and the zero at $x=b$ being simple, then a solution $w_{1}(x)$ is constructed having double zeros at $x=a$ and at $x=\alpha, \alpha<b$, and having exactly $n+3$ zeros on $[a, \alpha]$. Again, however, the impossibility of there existing a solution $z(x)$ of (1) having double zeros at $x=a$ and $x=\beta, \beta<\alpha$, with $n+3$ zeros on $[a, \beta]$ must be demonstrated. We restate Theorem 3.6 and establish the essential uniqueness of the solutions determining the conjugate points of $x=a$.

THEOREM. If there exists a solution $y(x)$ of (1) having at least $n+3$ zeros in $[a, \infty)$, there then exist $n$ points $\eta_{1}, \cdots, \eta_{n}\left(a<\eta_{1}<\cdots<\eta_{n}\right)$

Received by the editors October 7, 1970.

AMS 1969 subject classifications. Primary 3440, 3442.

Key words and phrases. Fourth order, selfadjoint differential equations, oscillation of solutions, conjugate points. 
and $n$ essentially unique solutions $y_{1}(x), \cdots, y_{n}(x)$ of (1) with the following properties:

(a) $y_{i}(x)$ has double zeros at $x=a$ and $x=\eta_{i}$;

(b) $y_{i}(x)$ has precisely $i+3$ zeros in $\left[a, \eta_{i}\right]$;

(c) any other solution $y(x)$ such that $y(a)=0$ has fewer than $i+3$ zeros in $\left[a, \eta_{i}\right]$.

Proof. As indicated above, we are concerned with (c) and the essential uniqueness of the solutions $y_{i}(x)$. Let $y(x)$ be a solution of (1) with exactly $n+3$ zeros, $n \geqq 1$, on $[a, b]$. We may assume by the results in [1] and our remarks above, that $y(x)$ has double zeros at $x=a$ and $x=b$ with $n-1$ simple zeros on $(a, b)$. Suppose there exists a solution $z(x)$ of (1) having double zeros at $x=a$ and $x=\beta, \beta<b$, and having $n-1$ simple zeros on $(a, \beta)$. For each of the solutions $y(x)$ and $z(x)$, we must have, by Lemma $2.1, y^{\prime \prime}(a) \cdot y^{\prime \prime \prime}(a)<0$ and $z^{\prime \prime}(a) \cdot z^{\prime \prime \prime}(a)$ $<0$. We assume, without loss of generality, that $y^{\prime \prime}(a) \cdot z^{\prime \prime}(a)>0$. By Theorem 3.3, the point $x=\beta$ must lie to the right of the last simple zero (if it exists), $x=c$, of $y$. As a result of Lemma 2.4, $y$ and $z$ are nonzero on $(b, \infty)$ and $(\beta, \infty)$, respectively. Moreover, since $y^{\prime \prime}(a) \cdot z^{\prime \prime}(a)>0$ and each of $y$ and $z$ has $n-1$ simple zeros followed by a double zero, we conclude that $y$ does not change sign on $(c, \infty)$ and that $y(\beta) \cdot z(b)>0$. Let $w(x)$ be the solution determined by the conditions $w(a)=w^{\prime}(a)=w^{\prime \prime \prime}(a)=0, w^{\prime \prime}(a)=1$. Then, by Lemma $2.1, w>0$ on $(a, \infty)$. Clearly each of $y$ and $z$ is independent of $w$. Moreover, it is easily established that $z(x)=d y(x)+k w(x)$. Solving for $d$ by calculating the third derivative at $x=a$, we find $d=z^{\prime \prime \prime}(a) / y^{\prime \prime \prime}(a)>0$. We assume, therefore, $d=1$. Now, consider the constant $k$. At $x=\beta$, we have $0=y(\beta)+k \cdot w(\beta)$ and thus $k=-y(\beta) / w(\beta)$. At $x=b$, we find $z(b)=[-y(\beta) / w(\beta)] w(b)$ implying $z(b) \cdot y(\beta)<0$ contradicting our result above and completing the proof of the theorem.

We remark, in conclusion, that many of the results of $\$ \$ 2$ and 3 of [1] can be extended to the general linear fourth order equation $y^{(4)}+p(x) y^{\prime \prime}+q(x) y^{\prime}+r(x) y=0$ when sign conditions are placed on $p, q$ and $r$ and on certain combinations of their derivatives [2].

\section{ReFERENCES}

1. W. Leighton and $Z$. Nehari, On the oscillation of solutions of self-adjoint linear differential equations of the fourth order, Trans. Amer. Math. Soc. 89 (1958), 325-377. MR 21 \#1429.

2. J. B. Scott, On the nature of solutions of the linear homogeneous fourth order differential equation, Ph.D. Dissertation, University of Houston, Houston, Texas, 1970.

University OF Houston, Houston, TeXas 77004 